\title{
Splenectomy versus Partial Splenic Embolization for Massive Splenomegaly Secondary to Hepatitis B-Related Liver Cirrhosis: A Case-Control Study
}

\author{
Shoufei Jiao, ${ }^{1}$ Hongxing Chen, ${ }^{2}$ Youlong Wang, ${ }^{2}$ Jiye Zhu, ${ }^{1}$ Jingwang Tan, ${ }^{2}$ and Jie Gao ${ }^{1}$ \\ ${ }^{1}$ Department of Hepatobiliary Surgery, Peking University People’s Hospital, Beijing 100044, China \\ ${ }^{2}$ Chinese PLA Medical School and Chinese PLA General Hospital, Beijing 100853, China \\ Correspondence should be addressed to Jingwang Tan; tanjingwang02@aliyun.com and Jie Gao; gaojie1@medmail.com.cn
}

Received 9 February 2016; Revised 23 May 2016; Accepted 31 May 2016

Academic Editor: Oliver Strobel

Copyright (C) 2016 Shoufei Jiao et al. This is an open access article distributed under the Creative Commons Attribution License, which permits unrestricted use, distribution, and reproduction in any medium, provided the original work is properly cited.

Background. Both splenectomy (SP) and partial splenic embolization (PSE) are used to treat massive splenomegaly (MSM) secondary to hepatitis B-related liver cirrhosis (HB-LC). This retrospective case-control study was conducted to compare the effects of SP and PSE on these patients. Methods. From July 2004 to January 2012, patients with MSM secondary to HB-LC who underwent SP or PSE were 1:1:1 matched with similar nonsurgery patients, respectively. Intraoperative situation, hematological indices, liver function, HBV DNA level, HBeAg seroconversion rate, morbidity, and mortality at 6 months postoperatively were compared. Results. Operative time, estimated blood loss, blood transfusion rate, severe pain, postoperative stay, and portal vein thrombosis (PVT) rate in the PSE group were significantly superior to the SP group, although SP and PSE were similar in liver function improvement, HBV suppression, morbidity, and mortality at 6 months postoperatively, and SP even improved WBC and PLT counts higher than PSE. Conclusion. Both SP and PSE are effective in improving liver function, increasing WBC and PLT counts, and suppressing replication of HBV for MSM secondary to HB-LC. Although postoperative improvement in WBC and PLT counts by SP can be higher than PSE, PSE is simple and minimally invasive and has a lower incidence of PVT.

\section{Introduction}

Hepatitis B is highly prevalent in China and frequently associated with liver cirrhosis and portal hypertension $(\mathrm{PH})$ that often cause splenomegaly $[1,2]$. SP is the most common surgical treatment for MSM secondary to HB-LC [3]. It has been generally agreed that SP is performed to control esophageal varices bleeding or as a modality in order to reverse severe thrombocytopenia and leukopenia. However, there are some risks associated with SP [4], such as hemorrhage, pulmonary atelectasis, pneumonia, pleural effusion, subphrenic abscess, gastric ileus, venous thrombosis, overwhelming postoperative infection (OPSI), and atherosclerosis. Alternatively, PSE could be an option that may have some advantages over SP in some instances [5]. Little is known about the comparison of SP and PSE on MSM secondary to HB-LC. This retrospective case-control study aimed to characterize the effects of PSE, in comparison with SP, on hematological indices, liver function, anti-hepatitis B virus, and PVT incidence in patients with MSM secondary to HB-LC.

\section{Methods}

2.1. Study Design. From July 2004 to January 2012, there were 1237 patients suffering from splenomegaly secondary to HB-LC who have been treated in our two institutes. Within these patients, 651 patients with severe esophageal varices, esophageal varices bleeding, refractory ascites, or liver cancer were initially excluded. Among the remaining 586 patients, 177 patients with mild hypersplenism (PLT > $6.0 \times 109 / \mathrm{L}$ ) were treated without surgery or interventional procedure, 148 patients underwent PSE, and 261 patients underwent SP. Within those without surgery or interventional procedure, 65 patients with enlarged spleen $(20-27 \mathrm{~cm}$ in craniocaudal length and $1000 \mathrm{~g}-2500 \mathrm{~g}$ in weight) were assigned 


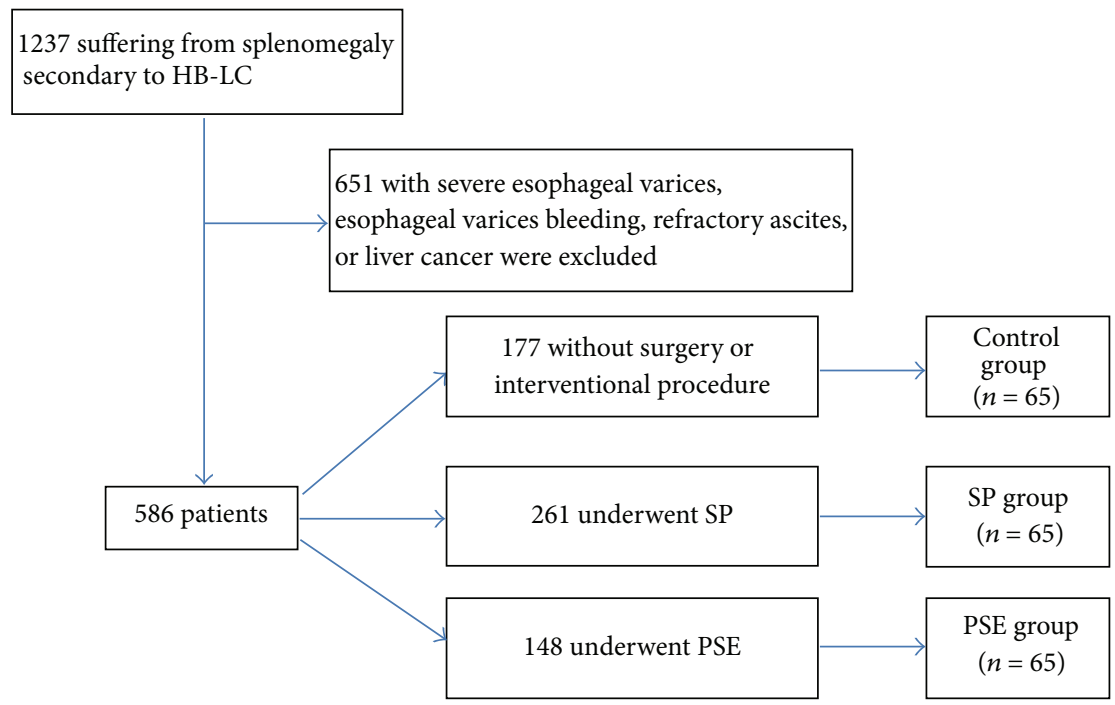

FIGURE 1: Flow diagram outlining the study design.

as the control group. Using a 1:1:1 case-control ratio, these 65 patients were randomly matched to patients who underwent PSE (PSE group) or SP (SP group) (Figure 1). The matching criteria included Child-Pugh grade, gender, age, serum HBV DNA level, antiviral therapy, spleen weight, esophageal varices degree, indocyanine green $15 \mathrm{~min}$ retention rate (ICG R15), comorbidities rate, and ASA grade.

All patients were subjected to detailed history, thorough physical examination, laboratory investigations (including bone marrow aspiration), abdominal ultrasonography, colorcoded duplex scanning of the portal circulation, upper gastrointestinal endoscopy, and abdominal computed tomography (CT) scans with oral and intravenous contrast and diagnosed by liver biopsy. All patients were provided with antiviral therapy if serum HBV DNA tests were positive. Liver-protective drugs were administered to patients with hepatic insufficiency. For splenomegaly, there were three treatment modalities: SP, PSE, or conservative treatment depending on PLT count and Child-Pugh grade with patients' consent. The spleen size was accurately measured before and after procedure by abdominal CT or ultrasound according to the recognized definition [6].

All patients gave informed consent and the study was authorized by the Hospital's Ethics Committee. Patient confidentiality was preserved according to the guidelines for studies of human subjects.

2.2. PSE Procedure. Under strict aseptic condition, PSE was performed according to a standard approach $[5,7]$. Briefly, the femoral artery was punctured by a 5.0 French catheter (Cook, Bloomington, USA; Terumo, Tokyo, Japan) via the Seldinger approach. Preliminary splenic arterial angiography was obtained to determine the configuration of splenic artery and the location of pancreatic branches. The tip of the catheter was placed as distal as possible at the hilus of the spleen in order to avoid ectopic embolization, and embolization was performed using embolic agent suspended in an antibiotic solution (gentamicin sulphate $16 \mathrm{mg}$ ) and contrast medium. The splenic infarction ratio was set at 50$70 \%$ [8]. During embolization, small amounts of contrast material were periodically injected through the catheter to monitor the flow distribution in the spleen. Immediately after each particle injection, postembolization angiography was performed and the infarction rate was calculated. Once a 50$70 \%$ ablation of splenic parenchyma was achieved, catheter was irrigated with saline and removed. The site of puncture was compressed for about 15 minutes.

Post-PSE supportive care included appropriate hydroelectrolytic infusion, systemic prophylaxis with intravenous antibiotics, cefoperazone $(1 \mathrm{~g} / 12 \mathrm{~h})$ for 5 days, and adapted analgesic treatment with nonsteroidal anti-inflammatory drugs or morphine. All patients remained in hospital until postembolization syndrome or any other significant complications disappeared. The precise infarction rate was calculated on CT examinations 2 weeks after PSE. Routine abdominal ultrasonography was performed at 30 day after PSE to exclude PVT.

The embolic agent used in this study was polyvinyl alcohol (PVA) in contour particles (Boston Scientific, Natick, MA, USA). Particle sizes are in the range of $355-500 \mu \mathrm{m}$ exclusively. Particles smaller than this range are not recommended considering the possibility of a more intense necrosis. For most of our patients, one vial of PVA was sufficient to embolize at least $50 \%$ of the spleen parenchyma.

2.3. SP Procedure. The operation was performed under general anesthesia with open or laparoscopy approach according to standard procedures $[3,9]$. All the operations were performed by the same experienced surgeon. SP was carried out immediately after opening and exploring the abdomen. In laparoscopic SP, the splenic artery was divided and ligated firstly in order to avoid massive hemorrhage. Subsequently, the splenic hilum was transected. Lateral position facilitates the dissection of perisplenic ligaments and hilus lienis structures due to gravity. Additionally, the operating table can be tilted to obtain appropriate patient's position at any time 
TABLE 1: Background characteristics of enrolled patients.

\begin{tabular}{|c|c|c|c|c|}
\hline Parameters & Control group $(n=65)$ & SP group $(n=65)$ & PSE group $(n=65)$ & $P$ value \\
\hline Child-Pugh grade $(\mathrm{A}: \mathrm{B}: \mathrm{C})$ & $54: 11: 0$ & $54: 11: 0$ & $54: 11: 0$ & 1.0000 \\
\hline Gender $(\mathrm{M}: \mathrm{F})$ & $43: 22$ & $45: 20$ & $44: 21$ & 0.9321 \\
\hline Age $(y)$ & $45.3 \pm 7.1$ & $46.1 \pm 8.7$ & $46.4 \pm 9.4$ & 0.7771 \\
\hline WBC count $\left(/ \mathrm{mm}^{3}\right)$ & $3.75 \pm 0.88$ & $2.81 \pm 1.02$ & $3.29 \pm 0.95$ & $<0.001$ \\
\hline PLT count $\left(10^{3} / \mathrm{mm}^{3}\right)$ & $7.3 \pm 2.5$ & $3.6 \pm 2.7$ & $4.5 \pm 2.4$ & $<0.001$ \\
\hline HBV DNA level $\left(\log _{10}\right)$ & $4.27 \pm 0.91$ & $4.16 \pm 0.85$ & $4.34 \pm 0.88$ & 0.5025 \\
\hline Antiviral protocol & & & & 0.9806 \\
\hline Lamivudine & 29 & 28 & 28 & \\
\hline Adefovir plus lamivudine & 6 & 5 & 5 & \\
\hline Entecavir plus lamivudine & 2 & 2 & 1 & \\
\hline Spleen weight $(\mathrm{g})$ & $1376 \pm 241$ & $1440 \pm 263$ & $1416 \pm 219$ & 0.3146 \\
\hline Esophageal varices degree (mild: no) & $21: 44$ & $24: 41$ & $26: 39$ & 0.6565 \\
\hline ICG R15 (\%) & $19.2 \pm 8.4$ & $18.4 \pm 9.1$ & $20.3 \pm 10.7$ & 0.5167 \\
\hline Comorbidities & 11 & 10 & 8 & 0.7531 \\
\hline Diabetes & 3 & 3 & 2 & \\
\hline Hypertension & 5 & 4 & 3 & \\
\hline COPD & 1 & 0 & 2 & \\
\hline Others & 2 & 3 & 1 & \\
\hline ASA grade $(\mathrm{I}: \mathrm{II}: \mathrm{III})$ & $41: 16: 8$ & $44: 17: 4$ & $38: 19: 8$ & 0.6793 \\
\hline
\end{tabular}

ICG R15, indocyanine green 15 min retention rate.

during surgery. The most common cause of convert from laparoscopic to open SP is unmanageable bleeding. Patients received pharmacologic or mechanical thromboprophylaxis after SP or PSE according to the risk for VTE and bleeding complications [10].

2.4. Antiviral Therapy and Follow-Up. Three groups were provided with standard antiviral agents, including lamivudine, entecavir, or adefovir. All patients were followed up at $1 \mathrm{~m}, 3 \mathrm{~m}$, and $6 \mathrm{~m}$ postoperatively. At each visit, a detailed history, physical examination, blood routine examination, liver function, serum HBV DNA level, HBeAg, ultrasound examination for PVT, and ICG R15 were obtained. If PVT was suspected in patients according to the routine ultrasound, clinical signs, or symptoms (such as abdominal distension, diarrhea, fever, abdominal discomfort, leukocytosis, and nausea), abdominal CT scans were subsequently performed to confirm this.

2.5. Statistical Analysis. Continuous variables were expressed as mean \pm standard deviation (SD) and analyzed using $t$ test. Qualitative variables were presented as number and percent. The chi-squared or Fischer's exact test was used for comparison between groups as appropriate. Statistical analyses were performed using SPSS software (version 13.0; SPSS Inc., Chicago, IL). A value of $P<0.05$ was considered statistically significant.

\section{Results}

3.1. Preoperative Characteristics and Operative Outcomes. Except for WBC and PLT counts, there were no significant differences in Child-Pugh grade, gender, age, serum
HBV DNA level, antiviral therapy, spleen weight, esophageal varices degree, ICG R15, comorbidity rate, and ASA grade between these three groups (Table $1, P>0.05$ ). Notably, operative time, estimated blood loss, blood transfusion rate, severe pain, postoperative stay, and PVT rate in the PSE group were significantly lower than the SP group $(P<0.05)$. Only one patient died of esophageal varices bleeding secondary to PVT in the SP group, and the total complication rate is similar between the SP and PSE groups (Table 2).

3.2. Hematological Indices. The preoperative and serial postoperative measurements of WBC and PLT counts are shown in Figure 2. Postoperative WBC and PLT counts rose higher in the SP and PSE groups compared with their preoperative results $(P<0.05)$, whereas there was no significant difference in the control group who have not received any drugs for improving WBC and PLT counts $(P>0.05)$. The postoperative increases of WBC and PLT counts in the SP group were higher than those in the PSE group $(P<0.05)$.

3.3. Liver Function. The levels of serum albumin, total bilirubin, international normalized ratio (INR), globulin, and ICG R15 before and after the procedures are summarized in Table 3 and Figure 3. At $3 \mathrm{~m}$ and $6 \mathrm{~m}$ postoperatively, patients in both SP and PSE groups had significantly increased albumin $(P<0.05)$ and sharply decreased TB, INR, and globulin $(P<$ 0.05). In contrast, these values in control patients remained unchanged comparing to preoperative levels $(P>0.05)$. Child-Pugh grade and ICG R15 at $6 \mathrm{~m}$ postoperatively were also improved significantly in the SP and PSE groups when compared with their preoperative state, as well as the values of the control group $(P<0.05)$. Overall, liver function 
TABLE 2: Intraoperative and postoperative results.

\begin{tabular}{|c|c|c|c|}
\hline Parameters & SP group $(n=65)$ & PSE group $(n=65)$ & $P$ value \\
\hline Operative time (min) & $107 \pm 12$ & $21.33 \pm 4.16$ & $<0.05$ \\
\hline Estimated blood loss (mL) & $610 \pm 136$ & 0.5 & $<0.001$ \\
\hline Rate of blood transfusion $[n(\%)]$ & $7(10.7 \%)$ & 0 & $<0.05$ \\
\hline Severe pain $[n(\%)]$ & $25(38.5 \%)$ & $9(13.8 \%)$ & $<0.05$ \\
\hline Complications [total (\%)] & $17(26.2 \%)$ & $15(23.1 \%)$ & 0.6839 \\
\hline Intra-abdominal abscess & 1 & 0 & \\
\hline Ascites & 10 & 6 & \\
\hline Internal bleeding & 1 & 0 & \\
\hline Left pleural effusion & 0 & 8 & \\
\hline Atelectasis & 4 & 1 & \\
\hline Pneumonia & 1 & 0 & \\
\hline Postoperative stay (d) & $10.6 \pm 2.3$ & $9.5 \pm 2.1$ & $<0.01$ \\
\hline PVT & $19(29.2 \%)$ & $2(3.0 \%)$ & $<0.001$ \\
\hline Mortality $[n(\%)]$ & $1(1.5 \%)$ & 0 & 1.0000 \\
\hline
\end{tabular}
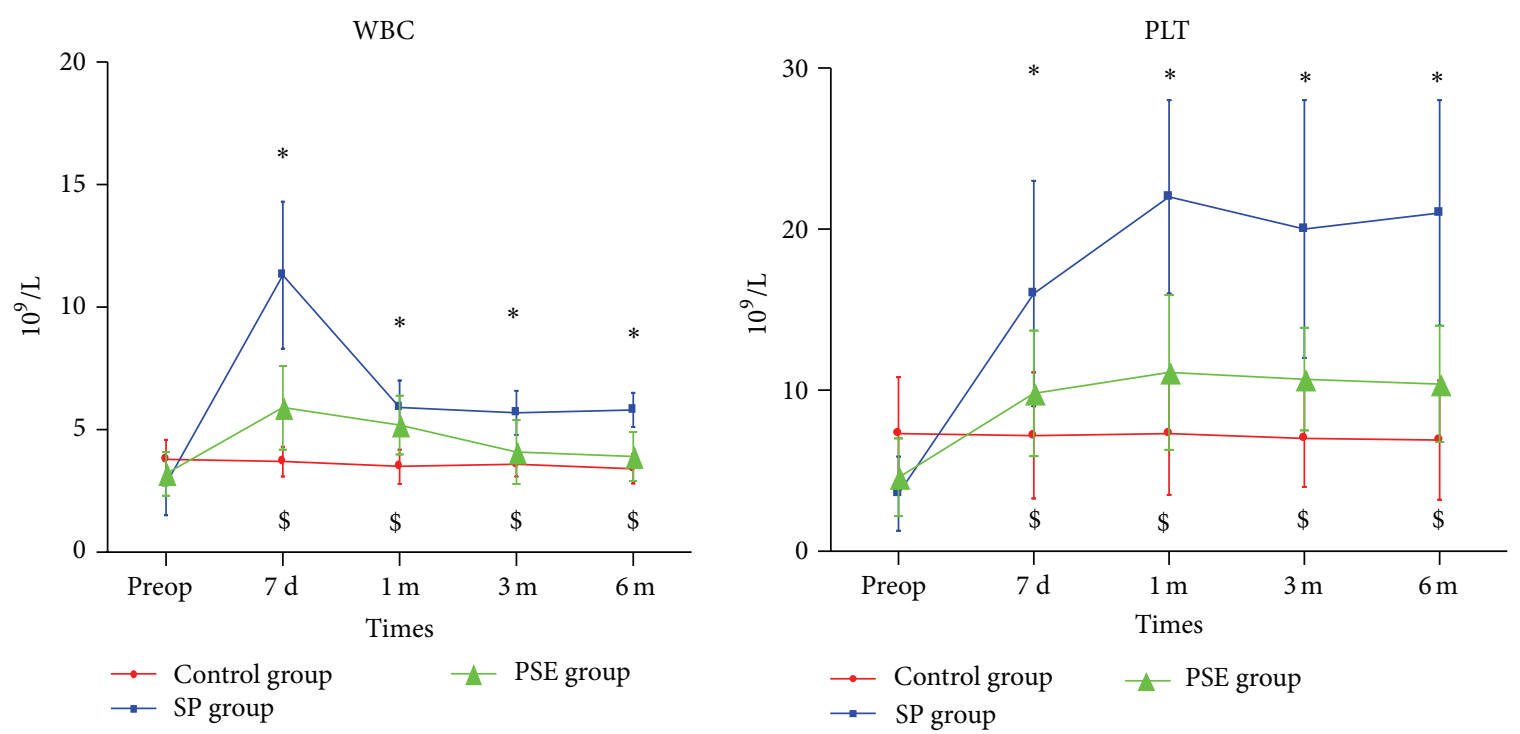

FIgURE 2: The preoperative and serial postoperative measurements of WBC and PLT counts. ( $*$ indicates significant difference compared with the baseline in the PSE group, $P<0.05 ; \$$ indicates significant difference compared with the baseline in the SP group, $P<0.05$.)

improvement was indistinguishable between the SP and PSE groups $(P>0.05)$.

3.4. HBV DNA Level and HBeAg Seroconversion Rate. HBV DNA levels and HBeAg seroconversion rates before and after the procedures are shown in Table 3. Postoperative HBV DNA levels decreased, and $\mathrm{HBeAg}$ seroconversion rates increased significantly in all patients $(P<0.05)$. Specifically, comparing to controls, HBV DNA levels have declined more in patients receiving SP or PSE $(P<0.05)$.

3.5. PVT. The incidences of PVT among the three groups are displayed in Table 3. The incidence of PVT in the SP group $(29.2 \%)$ was significantly higher than that in both control $(0 \%)$ and PSE groups $(3.0 \%)(P<0.05)$.

\section{Discussion}

4.1. Hematological Indices. Splenomegaly is a frequent finding in patients with HB-LC $[1,2,11]$. However, splenomegaly, even MSM secondary to HB-LC, is not considered as a surgical indication unless accompanied with severe hypersplenism or esophageal varices bleeding.

Hypersplenism may cause decreased blood cell counts mainly through five mechanisms [12]: (1) proportional decrease in peripheral blood cells due to the abnormal expansion of cell storage in the enlarged spleen; (2) enhanced phagocytosis of blood cells by monocyte and macrophage during hypersplenism; (3) myelosuppression induced by hepatitis B virus and hypersplenism; (4) autoantibody to blood cells produced by spleen; (5) esophageal varices bleeding that is often accompanied by hypersplenism. Accordingly, it 
TABLE 3: Short-term outcomes $(6 \mathrm{~m})$ among the three groups.

\begin{tabular}{lcccccc}
\hline Group & Time & HBV DNA level $\left(\log _{10}\right)$ & HBeAg positive $[n(\%)]$ & ICG R15 $(\%)$ & Child $(\mathrm{A}: \mathrm{B}: \mathrm{C})$ & PVT [ $n(\%)]$ \\
\hline \multirow{2}{*}{ Control group $(n=65)$} & Baseline & $4.27 \pm 0.91$ & $37 / 37(100 \%)$ & $19.2 \pm 8.4$ & $54: 11: 0$ & 0 \\
& After 6 m & $1.96 \pm 0.83^{*}$ & $29 / 37(78.4 \%)^{*}$ & $18.8 \pm 8.9$ & $55: 10: 0$ & 0 \\
\hline \multirow{2}{*}{ SP group $(n=65)$} & Baseline & $4.16 \pm 0.85$ & $35 / 35(100 \%)$ & $18.4 \pm 9.1$ & $54: 11: 0$ & 0 \\
& Postop $(6 \mathrm{~m})$ & $1.14 \pm 1.05^{* \#}$ & $24 / 35(68.6 \%)^{*}$ & $14.8 \pm 8.5^{* \#}$ & $62: 3: 0^{* \#}$ & $19(29.2 \%)^{* \#}$ \\
\hline \multirow{2}{*}{ PSE group $(n=65)$} & Baseline & $4.34 \pm 0.88$ & $34 / 34(100 \%)$ & $20.3 \pm 10.7$ & $54: 11: 0$ & 0 \\
& Postop $(6 \mathrm{~m})$ & $0.97 \pm 1.02^{* \#}$ & $21 / 34(61.7 \%)^{*}$ & $16.4 \pm 9.3^{* \#}$ & $63: 2: 0^{* \#}$ & $2(3.0 \%)^{\&}$ \\
\hline
\end{tabular}

${ }^{*} P<0.05$ is considered statistically significant compared with the baseline, ${ }^{\#} P<0.05$ is considered statistically significant compared with the control group, and ${ }^{\&} P<0.05$ is considered statistically significant compared with the SP group.
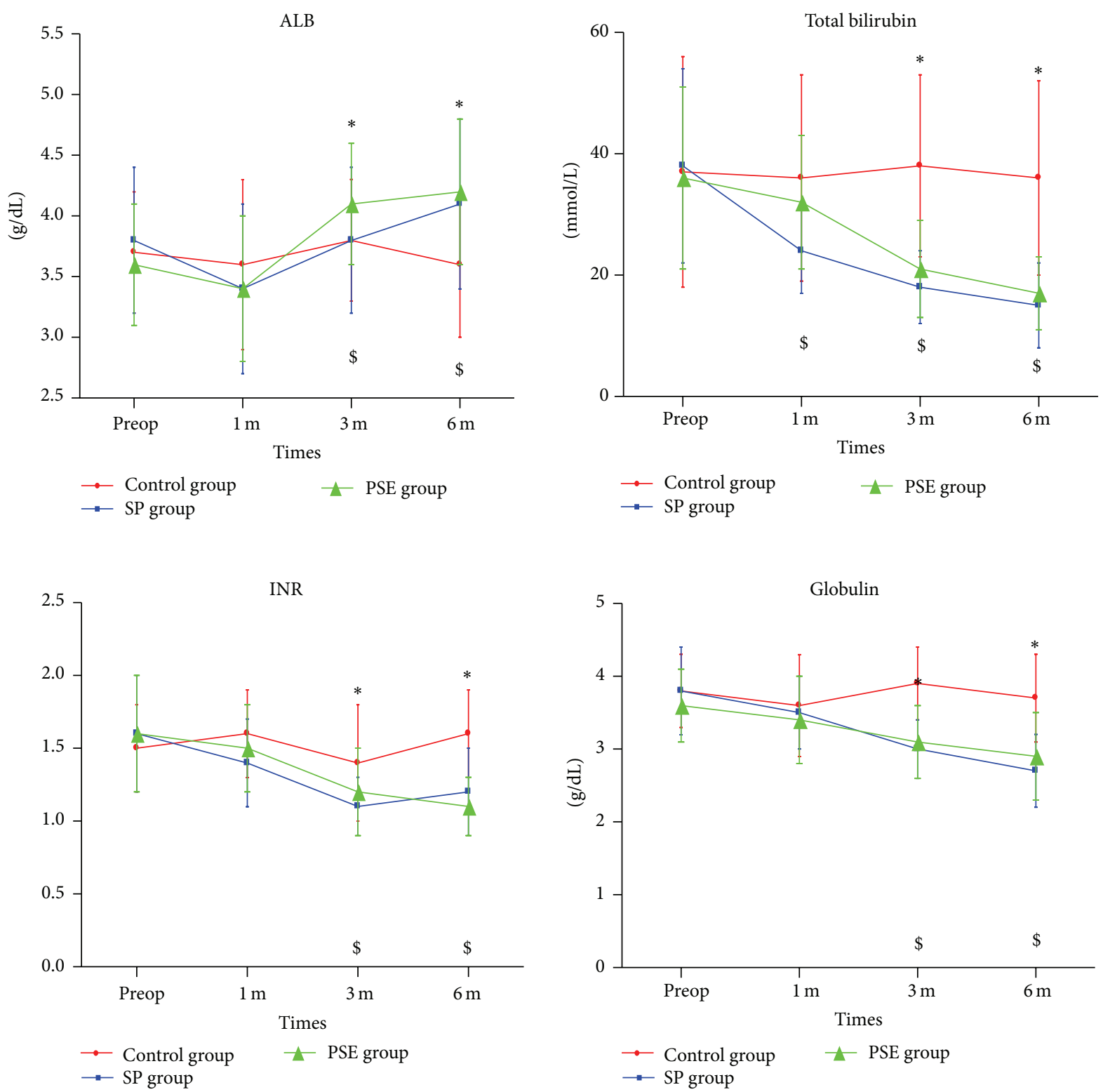

FIGURE 3: Serum albumin (ALB), total bilirubin, international normalized ratio (INR), and globulin before and after the procedure. (* indicates significant difference compared with the baseline in the PSE group, $P<0.05$; $\$$ indicates significant difference compared with the baseline in the SP group, $P<0.05$.) 
has been known that SP and PSE, both of which can lead to regression of splenomegaly, could raise WBC and PLT counts. Consistently in our study, postoperative WBC and PLT counts rose significantly in patients who underwent SP or PSE $(P<0.05)$. Interestingly, the increase is more obvious in SP-treated patients, which may be because of residual spleen parenchyma in PSE.

4.2. Liver Function. It has been well established that SP and PSE could alleviate portal vein pressure and improve liver function [13]. However, few studies have examined whether or not these effects may persist for more than six months. Ushitora et al. [14] have reported that SP and PSE could have a long-term effect on liver function, in which HB-LC patients were not evaluated. The assessments performed in our study suggest that SP and PSE have a lasting improvement in liver function also on HB-LC for at least six months.

The mechanism by which SP and PSE could improve liver function has not yet been elucidated. Decreasing portal vein pressure may be beneficial to prevent pathological bacterial translocation to the liver. In addition, the decrease of splenic vein flow leads to a compensatory increase of hepatic artery and mesenteric vein flow, resulting in more nutrientrich blood to the liver [15]. Moreover, an immunologic mechanism has been suggested by some studies $[14,16,17]$. For instance, it is possible that increased WBC could inhibit the replication of HBV and hence protect liver function, and antiviral therapy might indirectly contribute to the improvement of liver function as well.

4.3. HBV DNA Level and HBeAg Seroconversion Rate. Up to now, there are few studies concerning the impact of SP or PSE on HBV DNA level. We found that SP and PSE could reduce HBV DNA level, which may facilitate long-term improvement of liver function and possible prevention of disease progression. The nature and underlying mechanism of this intriguing finding is still unclear at the moment and certainly deserves further study in the future.

4.4. PVT. Previous studies have reported that the incidence of PVT after SP was between 8\% and 50\% [18]. On top of that, a large review of 37012 autopsies has shown that the odds of fatal pulmonary embolism in persons with SP were 5 -fold higher than matched controls who had not undergone SP [19]. Thomsen et al. [20] examined SP patients up to a year after surgery and identified that the adjusted relative risk of any venous thromboembolism in these patients is 3.4or 3.2-fold as the general population and appendectomized patients, respectively. The incidences of PVT in our study were $29.2 \%(19 / 65)$ after SP and 3.0\% (2/65) after PSE. It is possible that PVT after SP is associated with the increased amount and enhanced aggregation competence of platelet after SP. In addition, a higher plasma viscosity after SP can be attributed to increased blood cells and greater rigidity of erythrocytes [21]. The size of spleen before SP is likely to be associated with the incidence of PVT. Still blood in the stump of portal vein after SP also predisposes to PVT, which may explain the less PVT in PSE than SP [22].
In our study, there was one occurrence of PVT in a SP patient who died of gastrointestinal bleeding. Although most PVT remain asymptomatic and could recover well, this complication could be fatal if not timely treated, especially in the early stage. PVT after SP should be suspected whenever patients have abdominal pain, ileus, or fever. Color Doppler ultrasonography has a sensitivity of $93 \%$ and a specificity of $99 \%$ for diagnosing PVT, and routine Doppler ultrasonography after SP might be beneficial for early diagnosis. If diagnosed, prompt anticoagulation therapy can help to restore normal flow of portal vein [22]. Kawanaka et al. [23] have described the risk stratification of PVT after SP and reported that the administration of antithrombin III and danaparoid sodium could reduce the incidence of PVT.

4.5. OPSI. Besides PVT, OPSI is another complication worth noting associated with SP. The mortality rate of OPSI is $38-70 \%$ despite adequate treatment, and most deaths occur within $24 \mathrm{~h}-48 \mathrm{~h}$ [24]. Thus, the Society for Surgery of the Alimentary Tract recommended that SP can only be performed after trauma when blood loss volume was more than $1000 \mathrm{~mL}$, transfusion volume was more than 2 units, hemodynamics was unstable, or progressive hemorrhage was hinted. Otherwise, hemostatic, ligation and partial angioembolization of the splenic artery, simple splenorrhaphy, or partial SP was preferable [25]. When SP is compelled under certain circumstances, autotransplantation of splenic tissue in omental pockets might reserve sufficient splenic function [26]. It is estimated that at least $30 \mathrm{~mL}$ of splenic tissue is needed for return of splenic function after SP [27].

Compared with SP, several notable merits in PSE methods were documented in the present study: (1) PSE is minimally invasive, thus reducing operative time, blood loss, severe pain, and postoperative stay; (2) PSE has low incidence of PVT; (3) PSE preserves moderate splenic parenchyma to avoid OPSI; (4) PSE can also reverse hypersplenism, improve liver function, and even reduce HBV DNA level.

To our surprise, patients in the SP group experienced more severe pain than the PSE patients, which is different from the published data $[5,28]$. The distinct choices of drug regimen may lead to such unexpected results of pain perception in patients, since all PSE patients used nonsteroidal antiinflammatory drugs or morphine for analgesia immediately after PSE, while only a few SP patients volunteered to choose postoperative autocontrolled analgesic pump. As a result, only 9 of 65 PSE patients felt severe pain, and 25 of 65 PS patients felt severe pain.

Finally, the postoperative side-effects of PSE may include (1) postembolization syndrome, such as fever, nausea/vomiting, and spleen area pain; (2) ectopic embolization; (3) left pleural effusion; (4) splenic abscess; and (5) recurrence of MSM. Child-Pugh class $\mathrm{C}$ and splenic infarct volume $>540 \mathrm{~mL}$ are independent risk factors for complications after PSE. A partitioned and repeated PSE might therefore be a safer option in Child-Pugh class C patients to avoid the complications [29]. Although most complications after PSE can be alleviated by conservative treatment, percutaneous puncture and drainage of the abscess or SP is the final solution 
to splenic abscess. For patients with recurrent MSM, repeated PSE or SP is required.

\section{Conclusion}

In a summary, our study demonstrated that both SP and PSE could improve liver function, increase WBC and PLT counts, and suppress replication of $\mathrm{HBV}$ in patients with MSM secondary to HB-LC. These data indicate possible prevention of disease progression by both procedures. In comparison with SP that can raise more WBC and PLT counts, PSE is simple and minimally invasive and has a low incidence of PVT. PSE can be an effective option for patients who are not surgical candidates and for whom SP is contraindicated. Although our patient cohort was in a small number and mainly restricted to Child-Pugh class A, the findings suggest important differences between SP and PSE for MSM secondary to HB-LC. With longer follow-up and the additional measurement of portal vein pressure, a prospective randomized control trial based on our study would be potentially important to further identify the difference between SP and PSE with respect to liver function, replication of $\mathrm{HBV}$, and long-term prognosis.

\section{Competing Interests}

The authors declare that they have no conflict of interests.

\section{Authors' Contributions}

Jie Gao and Jingwang Tan contributed to this paper equally.

\section{Acknowledgments}

This study is supported by the National Natural Science Foundation (no. 81202342 and no. 81570590) and Graduate Student Scientific Research Funds of Peking University People's Hospital.

\section{References}

[1] X. Yue, H. Wang, F. Zhao et al., "Hepatitis B virus-induced calreticulin protein is involved in IFN resistance," The Journal of Immunology, vol. 189, no. 1, pp. 279-286, 2012.

[2] J. H. Hoofnagle, E. Doo, T. J. Liang, R. Fleischer, and A. S. F. Lok, "Management of hepatitis B: summary of a clinical research workshop," Hepatology, vol. 45, no. 4, pp. 1056-1075, 2007.

[3] X.-L. Zhan, Y. Ji, and Y.-D. Wang, "Laparoscopic splenectomy for hypersplenism secondary to liver cirrhosis and portal hypertension," World Journal of Gastroenterology, vol. 20, no. 19, pp. 5794-5800, 2014.

[4] E. P. Weledji, "Benefits and risks of splenectomy," International Journal of Surgery, vol. 12, no. 2, pp. 113-119, 2014.

[5] T. A. Hadduck, "Partial splenic artery embolization in cirrhotic patients," World Journal of Radiology, vol. 6, no. 5, pp. 160-168, 2014.

[6] P. M. Lamb, A. Lund, R. R. Kanagasabay, A. Martin, J. A. W. Webb, and R. H. Reznek, "Spleen size: how well do linear ultrasound measurements correlate with three-dimensional CT volume assessments?" British Journal of Radiology, vol. 75, no. 895, pp. 573-577, 2002.

[7] S. Kadir, Current Practice of Interventional Radiology, B. C. Decker Incorporated, 1991.

[8] K. Zhu, X. Meng, J. Qian et al., "Partial splenic embolization for hypersplenism in cirrhosis: a long-term outcome in 62 patients," Digestive and Liver Disease, vol. 41, no. 6, pp. 411-416, 2009.

[9] L. S. Feldman, "Laparoscopic splenectomy: standardized approach," World Journal of Surgery, vol. 35, no. 7, pp. 14871495, 2011.

[10] M. K. Gould, D. A. Garcia, S. M. Wren et al., "Prevention of VTE in nonorthopedic surgical patients : antithrombotic therapy and prevention of thrombosis, 9th ed: American College of Chest Physicians Evidence-Based Clinical Practice Guidelines," Chest, vol. 141, no. 2, supplement, pp. e227S-e277S, 2012.

[11] R. Orlando, F. Lirussi, S. M. M. Basso, and F. Lumachi, "Splenomegaly as risk factor of liver cirrhosis. A retrospective cohort study of 2,525 patients who underwent laparoscopy," In Vivo, vol. 25, no. 6, pp. 1009-1012, 2011.

[12] Y.-F. Lu, X.-Q. Li, X.-Y. Han, X.-G. Gong, and S.-W. Chang, "Peripheral blood cell variations in cirrhotic portal hypertension patients with hypersplenism," Asian Pacific Journal of Tropical Medicine, vol. 6, no. 8, pp. 663-666, 2013.

[13] D. Morihara, M. Kobayashi, K. Ikeda et al., "Effectiveness of combination therapy of splenectomy and long-term interferon in patients with hepatitis $C$ virus-related cirrhosis and thrombocytopenia," Hepatology Research, vol. 39, no. 5, pp. 439-447, 2009.

[14] Y. Ushitora, H. Tashiro, S. Takahashi et al., "Splenectomy in chronic hepatic disorders: portal vein thrombosis and improvement of liver function," Digestive Surgery, vol. 28, no. 1, pp. 9-14, 2011.

[15] O. Nishida, F. Moriyasu, T. Nakamura et al., "Interrelationship between splenic and superior mesenteric venous circulation manifested by transient splenic arterial occlusion using a balloon catheter," Hepatology, vol. 7, no. 3, pp. 442-446, 1987.

[16] Y. Arakawa, M. Shimada, H. Uchiyama et al., "Beneficial effects of splenectomy on massive hepatectomy model in rats," Hepatology Research, vol. 39, no. 4, pp. 391-397, 2009.

[17] T. Akahoshi, M. Hashizume, K. Tanoue et al., "Role of the spleen in liver fibrosis in rats may be mediated by transforming growth factor $\beta$-1," Journal of Gastroenterology and Hepatology, vol. 17, no. 1, pp. 59-65, 2002.

[18] E. R. Winslow, L. M. Brunt, J. A. Drebin, N. J. Soper, M. E. Klingensmith, and E. Dunn, "Portal vein thrombosis after splenectomy," American Journal of Surgery, vol. 184, no. 6, pp. 631-636, 2002.

[19] W. Pimpl, O. Dapunt, H. Kaindl, and J. Thalhamer, "Incidence of septic and thromboembolic-related deaths after splenectomy in adults," British Journal of Surgery, vol. 76, no. 5, pp. 517-521, 1989.

[20] R. W. Thomsen, W. M. Schoonen, D. K. Farkas, A. Riis, J. P. Fryzek, and H. T. Sørensen, "Risk of venous thromboembolism in splenectomized patients compared with the general population and appendectomized patients: a 10 -year nationwide cohort study," Journal of Thrombosis and Haemostasis, vol. 8, no. 6, pp. 1413-1416, 2010.

[21] D. A. F. Robertson, F. G. Simpson, and M. S. Losowsky, "Blood viscosity after splenectomy," British Medical Journal, vol. 283, no. 6291 , pp. $573-575,1981$. 
[22] M. Van 't Riet, J. W. A. Burger, J. M. Van Muiswinkel, G. Kazemier, M. R. Schipperus, and H. J. Bonjer, "Diagnosis and treatment of portal vein thrombosis following splenectomy," British Journal of Surgery, vol. 87, no. 9, pp. 1229-1233, 2000.

[23] H. Kawanaka, T. Akahoshi, S. Itoh et al., "Optimizing risk stratification in portal vein thrombosis after splenectomy and its primary prophylaxis with antithrombin III concentrates and danaparoid sodium in liver cirrhosis with portal hypertension," Journal of the American College of Surgeons, vol. 219, no. 5, pp. 865-874, 2014.

[24] P. D. Sinwar, "Overwhelming post splenectomy infection syndrome-review study," International Journal of Surgery, vol. 12, no. 12, pp. 1314-1316, 2014.

[25] "Surgical treatment of injuries and diseases of the spleen," Journal of Gastrointestinal Surgery, vol. 9, no. 3, pp. 453-454, 2005.

[26] R. D. Fremont and T. W. Rice, "Splenosis: a review," Southern Medical Journal, vol. 100, no. 6, pp. 589-593, 2007.

[27] G. R. Corazza, C. Tarozzi, D. Vaira, M. Frisoni, and G. Gasbarrini, "Return of splenic function after splenectomy: how much tissue is needed?" The British Medical Journal, vol. 289, no. 6449, pp. 861-864, 1984.

[28] T. Sakai, K. Shiraki, H. Inoue et al., "Complications of partial splenic embolization in cirrhotic patients," Digestive Diseases and Sciences, vol. 47, no. 2, pp. 388-391, 2002.

[29] H. Hayashi, T. Beppu, K. Okabe, T. Masuda, H. Okabe, and H. Baba, "Risk factors for complications after partial splenic embolization for liver cirrhosis," British Journal of Surgery, vol. 95, no. 6, pp. 744-750, 2008. 


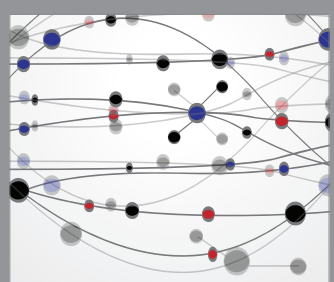

The Scientific World Journal
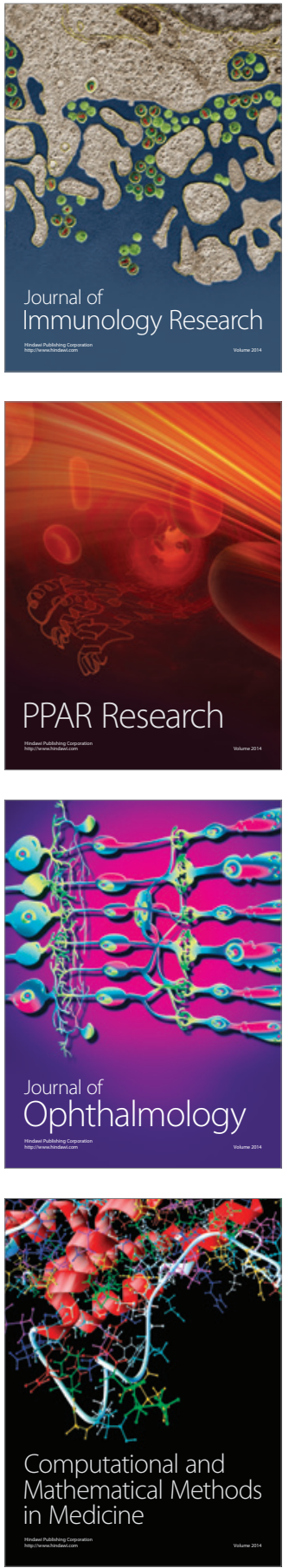

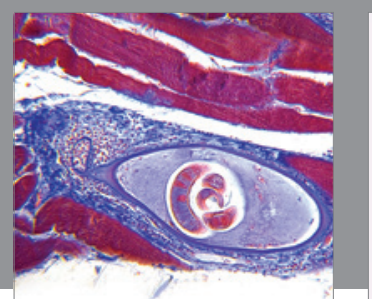

Gastroenterology Research and Practice

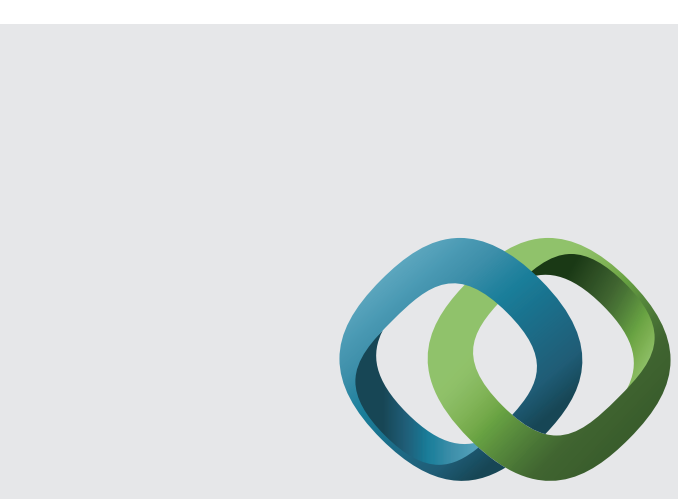

\section{Hindawi}

Submit your manuscripts at

http://www.hindawi.com
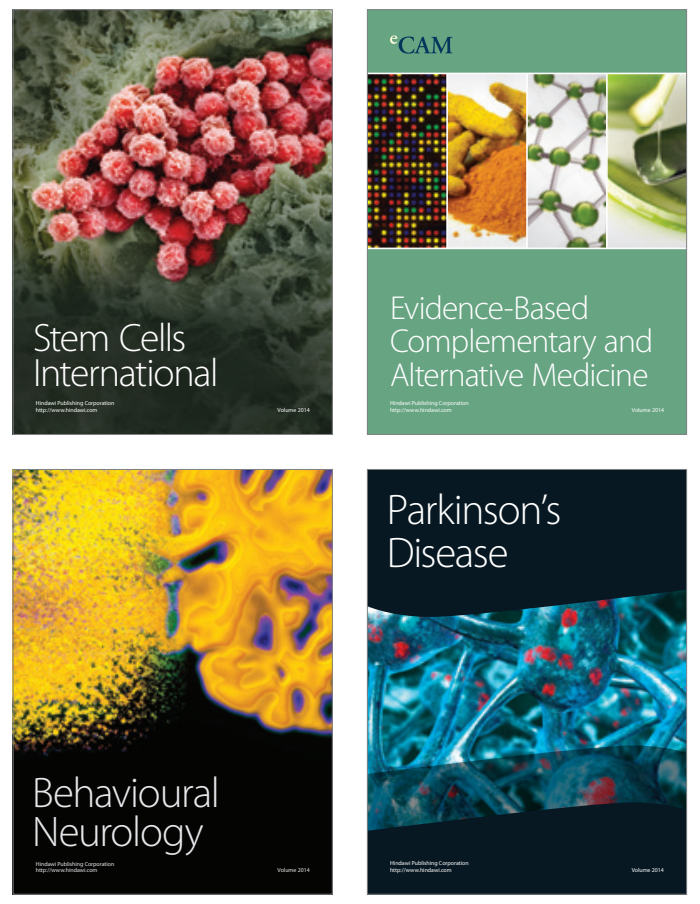
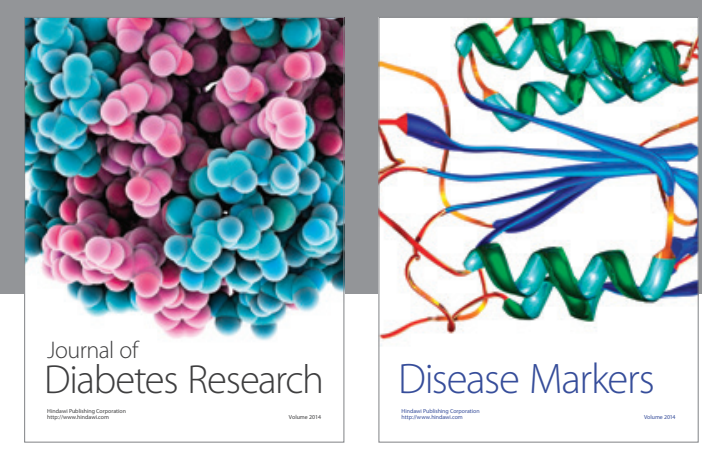

Disease Markers
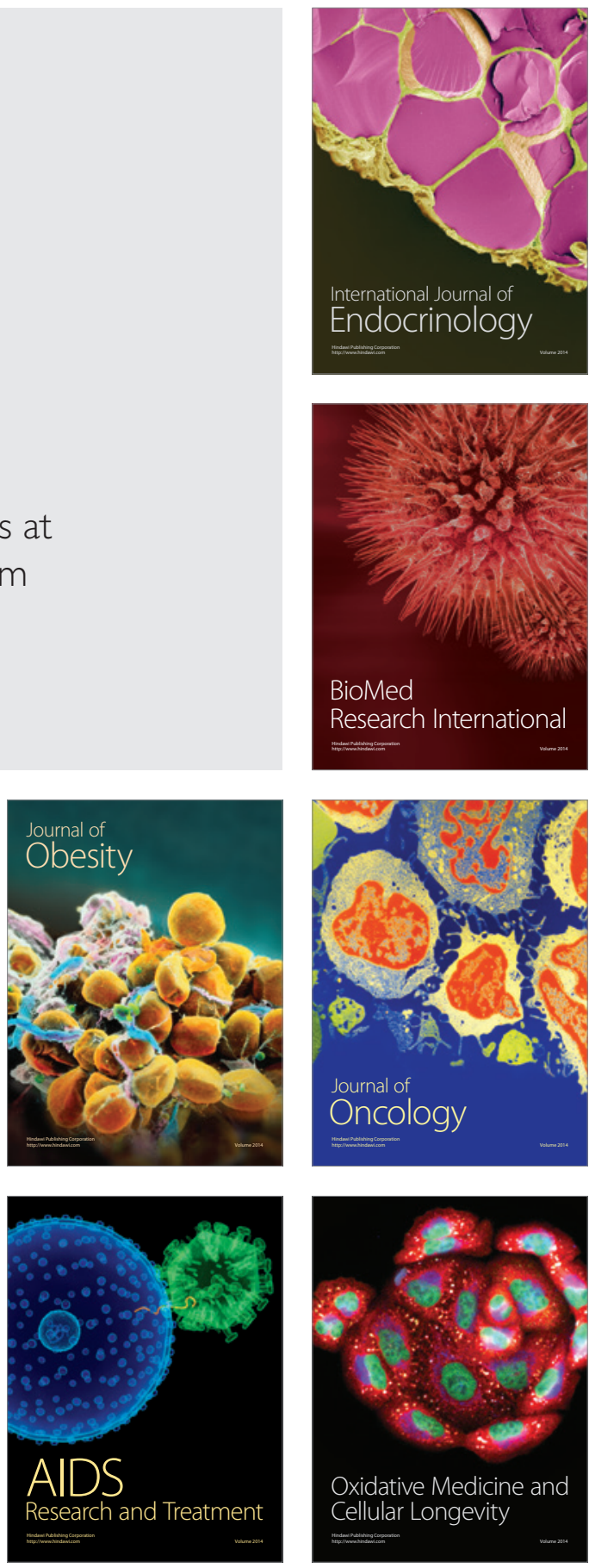